Check for updates

Cite this: RSC Adv., 2018, 8, 34726

Received 16th May 2018

Accepted 15th September 2018

DOI: $10.1039 / \mathrm{c} 8 \mathrm{ra0} 4156 \mathrm{c}$

rsc.li/rsc-advances

\section{Andrographolide-loaded silk fibroin nanoparticles $\uparrow$}

\author{
Xu Zhongyu, (D) Ren Jiangmeng, * Jing Qiufang, Ren Fuzheng, Huang Mengting, \\ Ding Wenrui and Zeng Bubing*
}

Andrographolide (AP) is a diterpenoid separated from Andrographis paniculata with a wide spectrum of biological activities including anti-inflammatory, anticancer, hepatoprotective, and antihyperlipidemic. However, its poor water solubility and instability result in lower bioavailability, which seriously limit its pharmacological function. In this study, the attempt to use regenerated silk fibroin (RSF) as a drug-carrier to encapsulate AP was reported. The AP-loaded RSF nanoparticles were prepared by a facile and clean method without any toxic agents. Moreover, special attention was paid to the optimization of formulation. Finally, the sizes of the AP-loaded RSF nanoparticles ranged from 200 to $1000 \mathrm{~nm}$, and the nanoparticles were spherically shaped, as seen by transmission electron microscopy. The drug loading and encapsulation efficiency were about $25.9 \%$ and $87.3 \%$, respectively. Furthermore, the release time of AP-loaded RSF nanoparticles was about 3 days. The particle size and drug release behaviour could be adjusted by treating with glycol amine. The in vitro cytotoxicity studies demonstrated that the RSF nanoparticles showed negligible cytotoxicity to cells, and the anti-proliferative activity of AP-loaded RSF nanoparticles showed that the AP-loaded RSF nanoparticles can adhere to Hela cells and MDA-MB-231 cells easily. All these results imply that this biomacromolecule drug nanocarrier has great potential for chemotherapy in clinical applications.

\section{Introduction}

Andrographolide (AP) is a natural diterpenoid separated from Andrographis paniculata and exhibits a wide spectrum of biological activities including anti-inflammatory, anticancer, ${ }^{1,2}$ hepatoprotective, ${ }^{3}$ and antihyperlipidemic. ${ }^{4}$ However, AP is insoluble in both water and nonpolar solvents. ${ }^{5}$ Furthermore, it is rapidly metabolized in the duodenum and jejunum to form a sulfate conjugate that is hydrophilic and likely to be impermeable. ${ }^{6}$ All these factors directly lead to lower oral bioavailability $(2.67 \%)$ of AP and seriously limit its pharmacological function. ${ }^{7}$ To solve these problems, researchers have designed many new dosage forms for AP such as dispersible tablets, ${ }^{8}$ dropping pills, ${ }^{9}$ oral microemulsion, ${ }^{10}$ liposomes, ${ }^{11}$ nanocrystals, ${ }^{12}$ and cyclodextrin inclusion complexes. ${ }^{13}$ Although desired results were obtained by improving the bioavailability of AP, the clinical application of AP is still restricted because of the lower drug-loading rate, entrapment rate, poor stability of pharmaceutical dosage forms, and the presence of organic solvent residue. To overcome these problems, it is necessary to develop suitable drug carrier systems.

Regenerated silk fibroin (RSF) from Bombyx mori silk is one of the fibrous proteins widely used in biomedical and

East China University of Science and Technology, Meilong Road 130, Shanghai, China. E-mail: zengbb@ecust.edu.cn; renjm@ecust.edu.cn

$\dagger$ Electronic supplementary information (ESI) available. See DOI: $10.1039 / \mathrm{c} 8 \mathrm{ra} 04156 \mathrm{c}$ pharmaceutical research. It consists of large hydrophobic regions segregated by relatively short and more hydrophobic regions..$^{14}$ RSF becomes water-soluble by adopting a random coil and/or helical conformation, but it easily becomes water insoluble after a conformation transition to $\beta$-sheet induced by various factors such as organic solvents, shear force, sonication, increase in temperature, and change of $\mathrm{pH}$. This implies that RSF has the characteristics of self-assembly, providing a unique opportunity in the design of supramolecular structures. In addition, silk fibroin exhibits good biocompatibility, controllable biodegradability and low inflammatory responses ${ }^{15}$ further suggesting that RSF is an ideal candidate as a nanoscale drug-delivery carrier.

Several efforts have been made to develop RSF nanoparticles (RSFNPs). For example, silk fibroin-albumin-blended nanoparticles with the size of $140-300 \mathrm{~nm}$ were prepared by the desolvation method and were utilized to load model drug methotrexate. ${ }^{16}$ In another study, ${ }^{17}$ cisplatin-loaded RSF nanoparticles approximately $59 \mathrm{~nm}$ in diameter prepared by electrospray demonstrated sustained in vitro release for more than 15 days. It was also reported that RSF nanoparticles can be prepared by simple dropwise addition of RSF solution into the water-miscible protonic and polar aprotonic organic solvents such as ethanol, methanol, $n$-propanol, isopropanol, tetrahydrofuran, and acetone. ${ }^{18-20}$ In this study, a clean method was developed to prepare RSFNPs carrying AP with predictable and controllable particle sizes based on the self-assembly of silk protein. To obtain uniform and stable nanoparticles, glycol 
amine $\left(\mathrm{mPEGNH}_{2}\right)$ was introduced in the processing, and special attention was paid to the function of $\mathrm{MPEGNH}_{2}$. The average particle size of the AP-loaded nanoparticles (APRSFNPs) was from 200 to $1000 \mathrm{~nm}$. The drug loading of APRSFNPs was about $25.7 \%$ which was much higher than previous results; the drug encapsulation efficiency was maintained at a suitable level of about $87.3 \%$, and the release time was about 3 days.

In this study, AP-RSFNPs were designed for applications in lymphatic chemotherapy, which is a relatively new therapeutic modality applied for the treatment of lymphatic metastases in patients with digestive tract and lung cancers. Unlike the conventional intravenous chemotherapy, the main approach to chemotherapy consists of the use of drug delivery system and local interstitial administrations such as intramuscular, subcutaneous, intratumoral and intraperitoneal injections for targeting agents to regional lymph nodes. Particles have been observed to pass through the lymphatic vessels but not through the blood capillaries mainly due to the difference in permeability. Studies ${ }^{21,22}$ on drug-loaded liposomes and PLGA particles show that the particle size ranging from several hundred nanometers to micrometers is good for lymphatic chemotherapy because the smaller particles pass through the lymph nodes easily, but the larger ones are easily entrapped by lymph node tissues during physical filtration, thus achieving long-time drug release.

\section{Experimental}

\section{Materials}

Cocoons of Bombyx mori silkworm were obtained from Zhejiang Province, China. AP was purchased from Sichuan Chengdu DESITE Biological Technology Co. Ltd. Glycol amine $\left(\mathrm{mPEGNH}_{2}\right)$ was purchased from Aladdin. The Hela line and MDA-MB-231 line were provided from Shanghai Institute of Cell Biology, Chinese Academy of Sciences. All the chemicals were purchased from Shanghai Zhenxin Chemical Factory (China).

\section{Preparation of RSF aqueous solution}

The RSF aqueous solution was prepared as previously reported ${ }^{23}$ with slight modifications. Briefly, Bombyx mori silkworm cocoons were cut into small pieces and boiled for 60 minutes in $0.5 \% \mathrm{Na}_{2} \mathrm{CO}_{3}$ solution; the solid was washed with deionized water several times. After drying completely, the degummed silk was dissolved in $9.3 \mathrm{~mol} \mathrm{~L}{ }^{-1} \mathrm{LiBr}$ solution at $60{ }^{\circ} \mathrm{C}$ for $4 \mathrm{~h}$. The solution was then dialyzed in a semipermeable cellulose tube (14 kD MWCO) against deionized water for 3 days to remove the salt. Afterward, the dialyzed silk fibroin solution was centrifuged at $10000 \mathrm{rpm}$ for 20 minutes. The supernatant was collected and then stored at $4{ }^{\circ} \mathrm{C}$ for further use. The final concentration of RSF solution was about 3-8 wt\%.

\section{Preparation of AP-loaded RSF nanoparticles}

The $10 \% \mathrm{mPEGNH}_{2}$ water solution was slowly added into RSF solution dropwise under gentle stirring at $100 \mathrm{rpm}$ and the final RSF concentrations of 5, 10, 20 and $30 \mathrm{mg} \mathrm{mL}^{-1}$ were obtained; then, the mixture was gently stirred for 30 minutes. Afterward, a certain amount of AP ethanol solution was slowly added into the mixture with different volume ratios of $V_{\mathrm{RSF}} / V_{\text {ethanol, as }}$ shown in Table 1 . The final mixture was gently stirred for 5 minutes and incubated in a refrigerator at $-20{ }^{\circ} \mathrm{C}$. After defrosting at room temperature, a milky emulsion or gelatin was obtained. To remove the non-encapsulated drug, $5 \mathrm{~mL}$ suspension was dialyzed with $1 \mathrm{~L}$ deionized water for $4 \mathrm{~h}$ and then lyophilized to obtain the lyophilization powder, which was stored at $4{ }^{\circ} \mathrm{C}$ for further use.

\section{Characterization of AP-loaded RSF nanoparticles}

Size analysis. The sizes of AP-loaded RSF nanoparticles were analyzed at room temperature with the PSS NICOMP particle sizing system.

Fourier transform infrared spectroscopy measurements. The Fourier transform infrared spectroscopy (FT-IR) measurements of pristine, AP-loaded RSF nanoparticles, PEG-treated AP-

Table 1 Characteristics of AP-loaded RSF nanoparticle suspension

\begin{tabular}{|c|c|c|c|c|c|c|c|}
\hline Entry & $\operatorname{RSF}(\%)$ & $\mathrm{AP}\left(\mathrm{mg} \mathrm{mL}^{-1}\right)$ & mPEGNH $_{2}(\%)$ & EtOH : RSF $(V / V)$ & Freezing time $(\mathrm{h})$ & Volume-mean diameter $(\mathrm{nm})$ & Variance (P.I.) \\
\hline 1 & 0.5 & 2 & $5 \%$ & 0.2 & 5 & 528.2 & 0.108 \\
\hline 2 & 0.5 & 4 & $10 \%$ & 0.25 & 10 & 423.9 & 0.002 \\
\hline 4 & 0.5 & 7 & $30 \%$ & 0.5 & 24 & 947.7 & 0.664 \\
\hline 5 & 1 & 2 & $10 \%$ & 0.4 & 24 & 322.8 & 0.097 \\
\hline 6 & 1 & 4 & $5 \%$ & 0.5 & 20 & 996.9 & 0.410 \\
\hline 9 & 2 & 2 & $20 \%$ & 0.5 & 10 & $>1000$ & - \\
\hline 10 & 2 & 4 & $30 \%$ & 0.4 & 5 & 238.9 & 0.312 \\
\hline 11 & 2 & 6 & $5 \%$ & 0.25 & 24 & 503.4 & 0.333 \\
\hline 12 & 2 & 7 & $10 \%$ & 0.2 & 20 & 893.9 & 0.254 \\
\hline 13 & 3 & 2 & $30 \%$ & 0.25 & 20 & 495.7 & 0.479 \\
\hline 14 & 3 & 4 & $20 \%$ & 0.2 & 24 & 495.7 & 0.479 \\
\hline
\end{tabular}


loaded RSF nanoparticles and the mixtures of AP and PEGtreated RSF nanoparticles as well as AP powder (in the form of attenuated total reflection (ATR)) were obtained with Shimadzu IRPrestige-21. For each measurement, 32 scans were collected with a resolution of $4 \mathrm{~cm}^{-1}$.

Morphology observations. The lyophilized powder was directly added on top of conductive tapes mounted on scanning electron microscopy (SEM) sample stubs. The morphologies of RSF nanoparticles were obtained with Hitachi S-3400N SEM at $15 \mathrm{kV}$. The as-prepared emulsion was diluted with de-ionized water before transmission electron microscopy (TEM) observation. TEM images were obtained with JEM-1400 at $200 \mathrm{kV}$.

Encapsulation and in vitro release of AP. The amount of AP in the nanoparticles was determined with Agilent HPLC 1260 after treating with methanol by ultrasound for $15 \mathrm{~min}$. HPLC systems were run on a C18 column $(4.6 \times 250 \mathrm{~mm}, 5 \mu \mathrm{m})$ at a column temperature of $30^{\circ} \mathrm{C}$ by employing a mobile phase of methanol and water delivered at a flow rate of $0.5 \mathrm{~mL} \mathrm{~min}{ }^{-1}$, and detection was conducted at $230 \mathrm{~nm}$. The injection volume was $20 \mu \mathrm{L}$. The calibration curve was linear for AP in the concentration range of $10-300 \mathrm{ng} \mu \mathrm{L}^{-1}\left(R^{2}=0.9996\right)$ with the linear equation $A=79.58 C-273.16$ ( $A$ is the peak area and $C$ is the concentration of the standard with the unit of $\left.n g \mu \mathrm{L}^{-1}\right)$. The relative standard deviation (RSD) values of inter- and intraday precision did not exceed 3\%. Data analysis and processing were carried out by Agilent Chemstation.

The drug loading capacity (DL) and encapsulation efficiency (EE) were calculated as follows:

$$
\begin{aligned}
& \mathrm{DL}(\%)=\frac{\text { weight of the drug in NPs }}{\text { weight of the NPs }} \times 100 \% \\
& \mathrm{EE}(\%)=\frac{\text { weight of the drug in NPs }}{\text { weight of the feeding drug }} \times 100 \%
\end{aligned}
$$

The in vitro release of AP from the drug-loaded AP nanoparticles and the influence of PEG. The release of three kinds of AP-RSFNPs, AP-saturated solution, and AP suspension was performed by the dialysis method in a phosphate buffer solution (PBS) maintained at $\mathrm{pH}$ 7.4. We placed one milliliter of AP-RSFNP suspension (0.9 $\mathrm{mg} \mathrm{mL} \mathrm{m}^{-1}$ in PBS), AP-saturated solution (0.043 $\mathrm{mg} \mathrm{mL}^{-1}$ in PBS), and AP suspension (0.9 $\mathrm{mg} \mathrm{mL}^{-1}$ in PBS) into dialysis bags. The bags were placed in a flask with $49 \mathrm{~mL}$ PBS being added as a dissolution medium. The conical flask was placed at $37{ }^{\circ} \mathrm{C}$ at the oscillation speed of $100 \mathrm{rpm}$. At regular time intervals, an aliquot of the dissolution medium $(1 \mathrm{~mL})$ was withdrawn and centrifuged (10 $000 \mathrm{rpm}$ for $5 \mathrm{~min}$ ); the supernatant was used for HPLC analysis. Then, the same volume of a fresh dissolution medium was replenished to maintain a sink condition. All operations were carried out in triplicates.

In vitro cytotoxicity assay with MTT. Hela cells were grown in culture media DMEM and seeded in 96-well plates at a density of 8000 cells per well at $37{ }^{\circ} \mathrm{C}$ under $5 \% \mathrm{CO}_{2}$. After $12 \mathrm{~h}$, the culture media were removed and replaced with fresh medium containing different amounts of pristine RSFNPs. After 24 h, 20 $\mu \mathrm{L}$ of MTT dye solution was added to each well and incubated for an additional $4 \mathrm{~h}$. Then, the medium was removed, and the formazan crystals were dissolved with $200 \mu \mathrm{L}$ of DMSO. An enzyme-linked immunosorbent assay (ELISA) reader was then used to measure the luminescence intensity at $570 \mathrm{~nm}$ of each well. The relative cell viability related to the control sample that was incubated with cell culture medium without RSF nanoparticles was calculated as follows:

$$
\text { Cell viability }=\frac{[A]_{\text {test }}}{[A]_{\text {control }}} \times 100 \%
$$

here, $[A]_{\text {test }}$ is the absorbance of the test sample and $[A]_{\text {control }}$ is the absorbance of the control sample.

The anti-proliferative activity of free AP and AP-RSFNPs. The anti-proliferative activities of free AP and AP-RSFNPs were evaluated by the MTT assay using Hela cells and MDA-MB-231 cells. The procedure was similar to that for the in vitro cytotoxicity described above; we just replaced RSFNPs with AP-RSFNPs. As a control group, Hela cells and MDA-MB-231 cells were also incubated with pure AP solutions, in which the amount of AP was set at the same level in the corresponding AP-RSFNPs.

\section{Results and discussion}

\section{Formation and characterization of AP-RSFNPs}

AP-RSFNPs with controllable sizes were obtained on the selfassembly of silk fibroin by adding ethanol into the RSF solution and then subjecting to freezing. Although the as-prepared AP-loaded RSFNP emulsion was very stable, which can be stored at room temperature for at least 4 weeks without apparent aggregation, it was found that the nanoparticles did not easily re-disperse in buffer solution after they were centrifuged from the emulsion; also, there were still a few large particles having sizes greater than $1000 \mathrm{~nm}$ (Fig. 1).

To increase the redispersibility and stability of AP-loaded RSF nanoparticles in the buffer solution and to prevent the formation of larger particles, mPEG- $\mathrm{NH}_{2}$ was introduced to the RSF nanoparticles. It was suggested that the interaction between RSF and
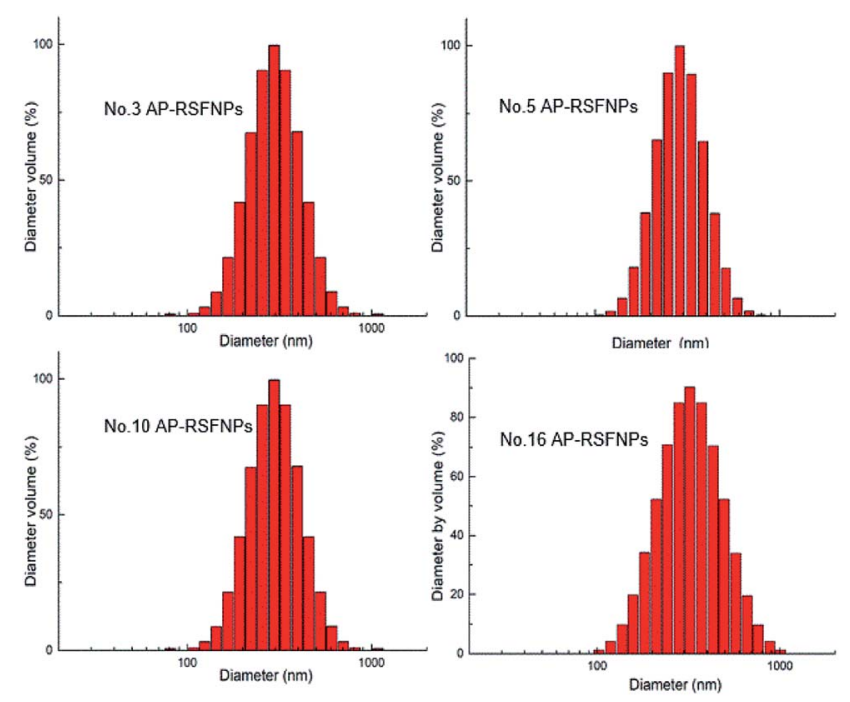

Fig. 1 AP-loaded RSF nanoparticle sizing distribution with the volume ratio between ethanol and RSF of 0.4 . 
PEG is mainly the electrostatic force between the negative charges on RSF and the positive charges on mPEG- $\mathrm{NH}_{2}$. The main reason for the improvement in the uniformity of the redispersed nanoparticles by such PEGylation was thought to be the steric repulsion effects of the tethered PEG strands attached on the surface of nanoparticles, as reported in the literature. ${ }^{\mathbf{2 4 , 2 5}}$

The initial RSF concentration, the AP concentration, the volume ratio between ethanol solution and RSF, the mass ratio between $\mathrm{MPEGNH}_{2}$ and RSF, and the freezing time affect the shape and size of particles. To obtain a suitable system, orthogonal experiments were designed, as shown in Table 1. Due to the increase in RSF concentration, the particles became smaller, and there was an increase in larger particles at the same time, as shown in Table 1. The increase in the AP concentration also affected the particle size. Suitable particles with an average size of $200-400 \mathrm{~nm}$ were obtained with the volume ratio between ethanol solution and RSF of 0.4. At the same time, when the volume ratio between ethanol solution and $\mathrm{RSF}$ reached 0.5 , the particle size increased to over $1000 \mathrm{~nm}$. We were able to control the AP-loaded RSF nanoparticles with particle sizes from 200 to $1000 \mathrm{~nm}$, which is suitable for lymphatic chemo-therapy, by changing the reaction conditions.

Both SEM and TEM images (Fig. 2 and 3) demonstrated that AP-RSFNPs were spherical granules without apparent aggregation. For instance, the particle size of nanoparticles shown in Fig. 2 was from 100 to $500 \mathrm{~nm}$, which was in accordance with the difference shown in their hydrodynamic diameters. To observe the effect of PEGylation, the TEM images of RSFNPS without PEG treatment and two kinds of PEG-treated RSFPNPs, which were treated with PEG before nanoparticle formation (BPEG-RSFNPs), after nanoparticle formation (A-PEG-RSFNPs), and their corresponding drug loading particles AP-RSFNPs, BPEG-AP-RSFNPS and A-PEG-AP-RSNPs are shown in Fig. 2. After loading the drug, the particles were clearly larger than those without drug loading, but their sizes were still smaller than $500 \mathrm{~nm}$. The particle distribution of PEG-treated particles tended to be better than that of the ones without PEG treatment, which confirmed the findings of particle analysis. There was no clear change between B-PEG-RSFNPs and A-PEG-RSFNPs. Fig. 3 shows the SEM images of four kinds of AP-RSFNPs, which were
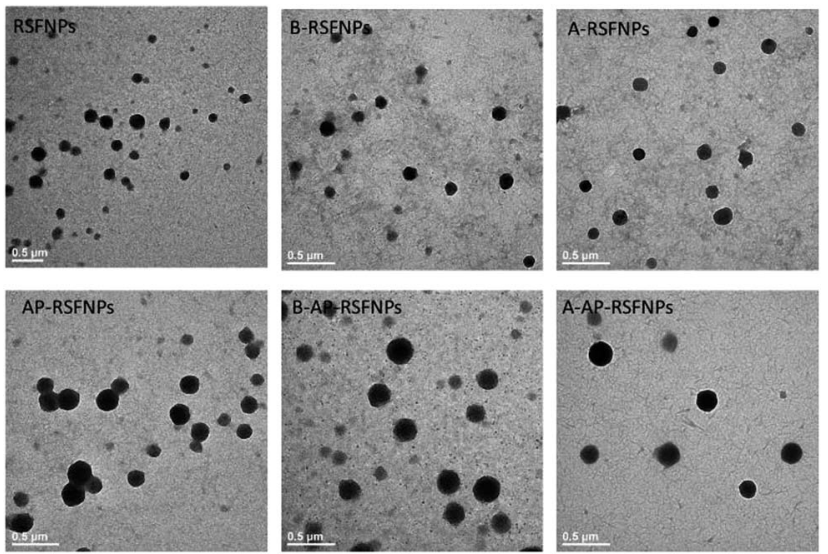

Fig. 2 TEM image of RSF nanoparticle system.
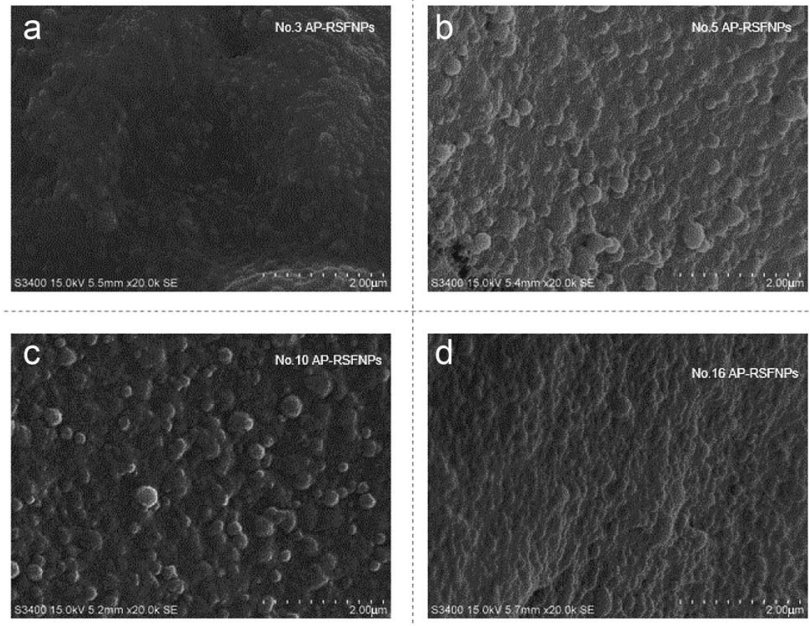

Fig. 3 SEM image of the AP-loaded RSF nanoparticle system with the volume ratio between ethanol and RSF of 0.4 ((a): no. 3; (b): no. 5; (c): no. 10; (d): no. 16).

prepared with the volume ratio of ethanol and RSF of 0.4; the SEM images also agreed with the findings of particles analysis.

The structure of silk fibroin consists of large regions of hydrophobic amino acids separated by short hydrophilic regions. The hydrophobic domain of silk fibroin consists of largely repeated sequences of (Gly-Ala-Gly-Ala-Gly-Ser) ${ }_{n}$. Silk fibroin shows characteristic vibration bands between 1630 and $1650 \mathrm{~cm}^{-1}$ for amide I ( $=\mathrm{O}$ stretching), $1540-1520 \mathrm{~cm}^{-1}$ for amide II (secondary $\mathrm{N}-\mathrm{H}$ bending) and $1270-1230 \mathrm{~cm}^{-1}$ for amide III (C-N and $\mathrm{N}-\mathrm{H}$ functionalities) in the FT-IR spectra. In native silk fibers, the repeated sequences organize together by hydrogen bonds, forming $\beta$-sheet, crosslinking and stabilized silk structures. RSF commonly exhibits $\alpha$-helices and random coil instead of a $\beta$-sheet structure in aqueous solution. Since the FT-IR spectra of $\alpha$-helices, random coil and $\beta$-sheet are different, ${ }^{26}$ the FT-IR spectra of different RSF particles were recorded to explore the conformation of RSF in these particles.

To further investigate the effect of PEG, we obtained the infrared spectra of RSFNPs (RSFNPs1), B-PEG-RSFNPS (RSFNPs2) and A-PEG-RSNPs(RSFNPs3) and the corresponding drug loaded particles RSFNPs4, RSFNPs5 and RSFNPs6, as shown in Table 2.

Table 2 Infrared spectroscopy data of different RSF nanoparticles, pristine RSF lyophilization powder, original materials and their physical mixture powder

\begin{tabular}{llll}
\hline Material & Amides I $\left(\mathrm{cm}^{-1}\right)$ & Amides II $\left(\mathrm{cm}^{-1}\right)$ & Amides III $\left(\mathrm{cm}^{-1}\right)$ \\
\hline RSFNPs1 & 1624.06 & 1523.76 & 1263.37 \\
RSFNPs2 & 1625.99 & 1529.55 & 1219.01 \\
RSFNPs3 & 1620.21 & 1529.55 & 1222.03 \\
RSFNPs4 & 1629.85 & 1517.98 & 1232.51 \\
RSFNPs5 & 1620.21 & 1514.12 & 1230.58 \\
RSFNPs6 & 1624.06 & 1514.12 & 1234.44 \\
RSF & 1641.42 & 1529.22 & 1238.50 \\
mPEGNH 2 & - & - & 1240.23 \\
AP & 1674.21 & - & - \\
Physical & 1641.42 & 1525.69 & $1220.94,1240.43$
\end{tabular}


The infrared spectral data of each material in the system and the physical mixtures were also studied for comparison.

The pristine RSF lyophilized powder (RSF) exhibited absorption peaks at $1641 \mathrm{~cm}^{-1}$ (amide I), $1529 \mathrm{~cm}^{-1}$ (amide II), and $1238 \mathrm{~cm}^{-1}$ (amide III), which represent the $\alpha$-helices and random coil configuration of RSF in aqueous solution. RSFNPs1 exhibited absorption peaks at $1624 \mathrm{~cm}^{-1}$ (amide I), $1524 \mathrm{~cm}^{-1}$ (amide II) and $1263 \mathrm{~cm}^{-1}$ (amide III), which represent the $\beta$ sheets. After comparing the FT-IR spectral data of RSFNPs1, RSFNPs2 and RSFNPs3, it was found that their amide II and amide III were different, which showed that treating with PEG can partly reduce the fractions of $\beta$-sheets; this reduction was slightly higher in particles synthesized by PEG treatment before self-assembly compared with that for PEG treatment after selfassembly. The schematic representation of the silk fibroin self-assembly with PEGylation process is shown in Fig. 4. First, when RSF was mixed with PEG, electrostatic interaction was formed between the negative charges on RSF and the positive charges on mPEG- $\mathrm{NH}_{2}$. During the process of self-assembly, this force and the steric repulsion effects of the tethered PEG strands may cause a decrease of $\beta$-sheet domains in RSFNPs; after self-assembly, the other part of PEG was used to modify the surface of the particles. Without PEG treatment, more $\beta$-sheets were transformed from $\alpha$-helices and random coil of RSF, which was the cause of the formation of larger particles.

\section{Drug loading, encapsulation efficiency and in vitro drug release of AP-RSFNPS}

The drug loading capacity of AP-RSFNPs increased with increasing initial AP concentration. In the meantime, the drug loading capacity also increased when the $\mathrm{m}_{\mathrm{AP}} / \mathrm{m}_{\mathrm{RSF}}$ ratio increased, but the extent of improvement was not significant and the solution of AP in ethanol was limited. Different PEG treatment processes can also change the encapsulation efficiency, as shown in Table 3. The drug loading and encapsulation efficiency of AP-RSFNPs2 (PEG treatment before encapsulation), AP-RSFNPs3(PEG treatment after encapsulation), and AP-RSFNPs4 (PEG treatment before and after) were better than those of AP-RSFNPs1 (without PEG treatment). After comparing three types of PEG-treated drug loading particles APRSFNPs2, AP-RSFNPs3 and AP-RSFNPs4, we inferred that the

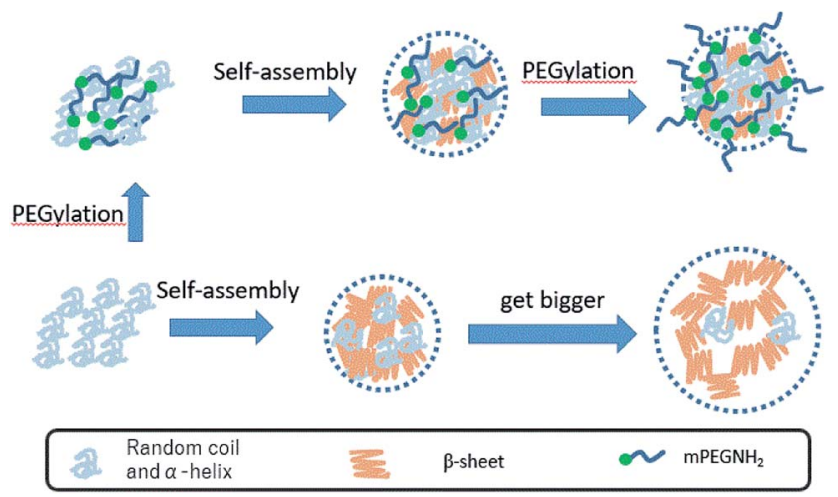

Fig. 4 Schematic representation of the silk fibroin self-assembly with PEGylation process.
Table 3 Drug loading and encapsulation efficiency of different nanoparticles

\begin{tabular}{lcc}
\hline Sample & EE\% & DL\% \\
\hline AP-RSFNPs1 & 84.5 & 28.9 \\
AP-RSFNPs2 & 89.6 & 26.4 \\
AP-RSFNPs3 & 85.7 & 25.5 \\
AP-RSFNP4 & 87.3 & 25.9
\end{tabular}

encapsulation efficiency of AP-RSFNPs2 was better than those of the other two. This increase may be caused by the solubilization effect of PEG in the silk fibroin water solution. With the PEG treatment, the drug loading decreased, whereas encapsulation efficiency increased. The suitable drug loading reached $25.9 \%$, and the encapsulation efficiency reached $87.3 \%$ with the PEG treatment before and after drug encapsulation when [RSF] $=$ $5 \mathrm{mg} \mathrm{mL}{ }^{-1}, m_{\mathrm{AP}} / m_{\mathrm{RSF}}=12: 25$ and $m_{\mathrm{PEGNH}_{2}}: m_{\mathrm{RSF}}=0.2: 1$.

It has been reported ${ }^{27}$ that the drug release from the RSF nanoparticles and microparticles depends on the silk structure and interactions between the compound and silk. The reduction of $\beta$-sheet domains in the particles allows faster degradation, which then speeds up the drug release. ${ }^{28}$ The $\beta$-sheet domains of different PEG-treated RSFNPs were in different levels, which was confirmed by FT-IR. The in vitro drug-release curves of AP-loaded RSFNPS are shown in Fig. 5. After comparing the two kinds of nanoparticles with AP suspension and AP-saturated solution, it was found that the release rate of B-AP-RSFNPs was slower than that from AP-saturated solution; however, it was faster than that from AP suspension and A-AP-RSFNPs. The release of B-APRSFNPs was similar to that of A-AP-RSFNPs in the initial $8 \mathrm{~h}$ and then became faster after $8 \mathrm{~h}$. For B-AP-RSFNPs, 90.9\% AP was released in $72 \mathrm{~h}$, whereas that of A-AP-RSFNPs was $65.8 \%$. These drug release results agreed with the findings of FT-IR and indicated that by changing PEG treatment methods we can control the $\beta$-sheet fraction in the particles, which can be used to design suitable kinds of AP-RSFNPs for different uses.

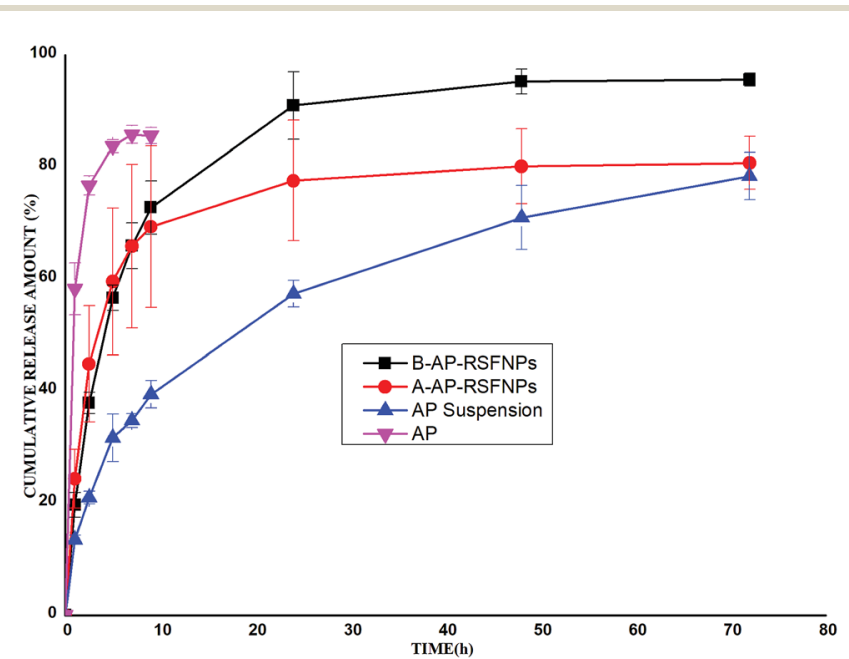

Fig. 5 In vitro release profiles of different B-AP-RSFNPs, A-APRSFNPs, AP (AP-saturated solution) and AP suspension determined by a dialysis method in PBS ( $\mathrm{pH}$ 7.4) as a release medium. Data represent mean \pm SD of three tests $(n=3)$. 


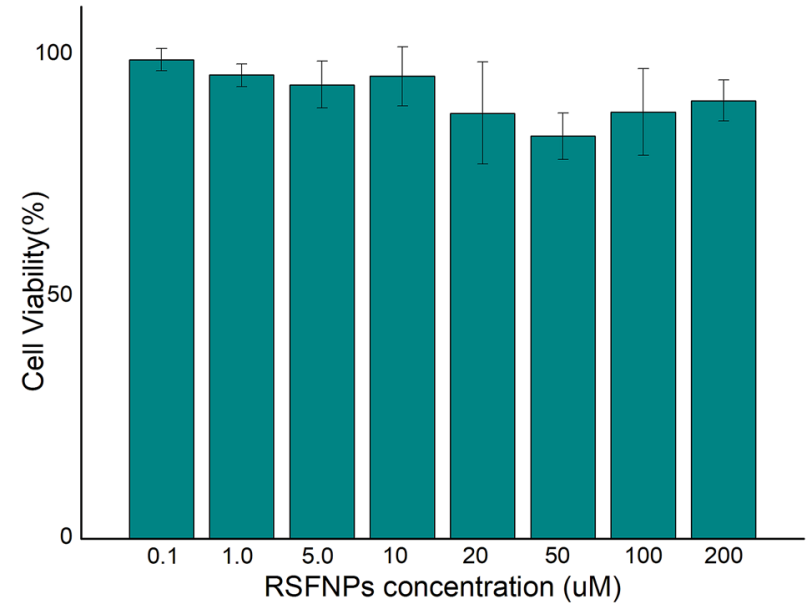

Fig. 6 Cytotoxicity of pristine RSFNPs with Hela cells after $24 \mathrm{~h}$ incubation.

\section{In vitro cytotoxicity of RSFNPS}

The cytotoxicity of pristine RSF nanoparticles was evaluated with Hela cells by MTT assay, and the results are shown in Fig. 6. Fig. 6 indicates that the cells incubated with RSF nanoparticles with low concentration $(0.1 \mu \mathrm{M})$ exhibited almost the same viability as that of the control. The cell viability decreased slowly with the increase in the concentration of RSF nanoparticles, but it was still about $80 \%$ when the concentration of RSF nanoparticles was as high as $200 \mu \mathrm{M}$. Thus, the cytotoxicity of RSF nanoparticles is quite low.

\section{In vitro cellular growth inhibition of Hela cells and MDA-MB- 231 cells with AP-RSFNPs}

The viability of Hela cells and MDA-MB-231 cells after contacting with AP-RSFNPs for $24 \mathrm{~h}$ is shown in Fig. 6 and 7. With the increase in the amount of AP-RSFNPs in the culture media, more Hela cells were killed or inhibited because more AP drugs were released. When the AP concentration in the culture medium was $50 \mu \mathrm{M}$, less than $10 \%$ Hela cells were alive after $24 \mathrm{~h}$ of incubation. To evaluate the anti-cancer efficiency of the

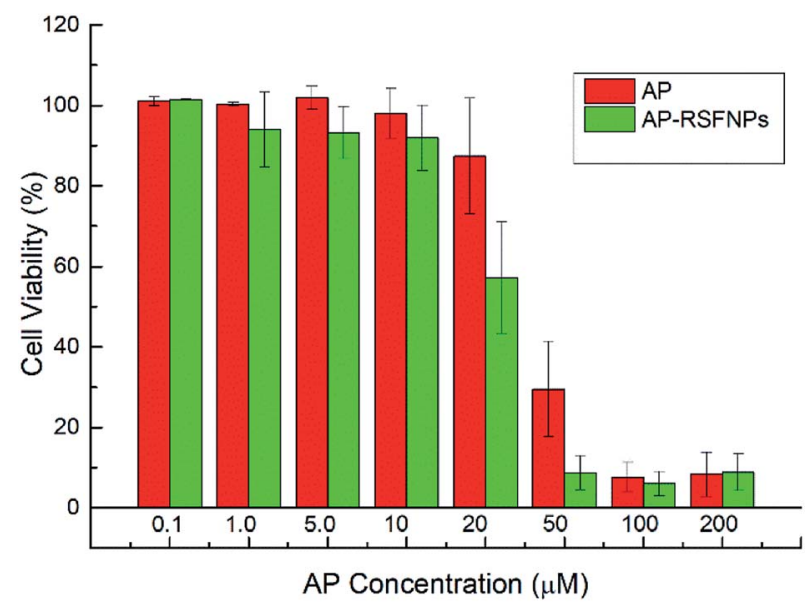

Fig. 7 Cell viability of AP and AP-RSFNPs with Hela cells after $24 \mathrm{~h}$ incubation.

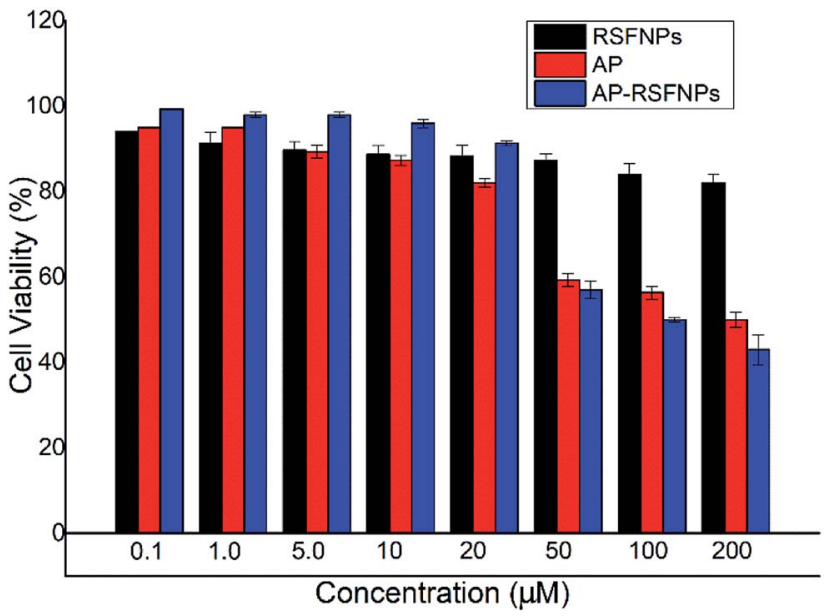

Fig. 8 Cell viability of RSFNPs, AP and AP-RSFNPs with MDA-MB-231 cells after $24 \mathrm{~h}$ incubation.

drug after encapsulating in RSFNPs, free AP drug was used as the control. Generally, more Hela cells were killed or inhibited in the AP-RSFNP groups than that in the control groups. There was no large difference when the AP concentration was lower than $20 \mu \mathrm{M}$, whereas the difference became greater when the AP concentration increased to over $20 \mu \mathrm{M}$. When the AP concentration increased to $50 \mu \mathrm{M}$, the viability of Hela cells contacting with AP-RSFNPs was $8.8 \%$, whereas that with free AP was $29.5 \%$. However, when the concentration increased to over $100 \mu \mathrm{M}$, the viability of Hela cells was almost the same in these two groups. Fig. 7 shows that contact with AP-RSFNPs in $50 \mu \mathrm{M}$ AP concentration has the same cell inhibition effect as that with free AP at $100 \mu \mathrm{M}$. Fig. 8 shows that when the AP concentration was lower than $20 \mu \mathrm{M}$, the MDA-MB-231 cells killed or inhibited in the AP-RSFNP groups were slightly less than that in the control groups. When the AP concentration was higher than 50 $\mu \mathrm{M}$, the cell viability of AP-RSFNPs was slightly higher than that of the control group.

Two kinds of cell experiments showed that the cell killing effect of AP-RSFNPs exhibited the same trend as that when the AP concentration was low; the cell killing effect of AP-RSFNPs was lower or equal than that in the control. When the AP concentration was higher, the killing effect was higher than that in the control group. The same trend indicated that AP-RSFNPs can adhere to Hela cells and MDA-MB-231 cells easily. At the same time, AP encapsulated in AP-RSFNPs required time to release, and not all AP released at $24 \mathrm{~h}$, which can be found from the release curves shown in Fig. 4. Due to the release of more AP from AP-RSFNPs, the advantage of such AP-RSFNPs is clear.

\section{Conclusions}

In this paper, we reported the preparation of a natural polymerbased nanocarrier to encapsulate a hydrophilic anti-cancer drug andrographolide and studied the preparing conditions and properties of the particles. The AP-loaded RSF nanoparticles were successfully prepared by a green and mild method, in which only additions of ethanol, mPEG- $\mathrm{NH}_{2}$ and freezing 
RSF-ethanol solution were involved. The characterization of nanoparticle system shows that AP-RSFNPs have controllable shape and size by the designed process. The particles have higher drug loading (25.9\%) and better encapsulation efficiency (87.3\%). The average volume size of such AP-RSFNPs is about $300 \mathrm{~nm}$, which could be beneficial for lymphatic chemotherapy. The in vitro release time of AP-loaded RSFNPs is about 3 days, and the release rate can be adjusted by the PEGylation process. The $24 \mathrm{~h}$ cellular growth inhibition of Hela cells with APRSFNPs is better than that with free AP. All these results imply that such an anti-cancer drug nanocarrier has great potential in future clinical treatments.

\section{Conflicts of interest}

There are no conflicts to declare.

\section{Acknowledgements}

This research was financially supported by the National Natural Science Foundation of China (21302053).

\section{Notes and references}

1 R. A. k. Sriram Rajagopal, D. S. Deevi, C. Satyanarayana and R. Raja gopalan, J. Exp. Ther. Oncol., 2003, 3, 147-158.

2 E. S. A. M. T. Islam, S. J. Uddin, M. A. Islam, S. Shaw, I. N. Khan, S. S. S. Saravi, S. Ahmad, S. Rehman, V. K. Gupta, M.-A. Gaman, A. M. Gaman, S. Yele, A. K. Das, J. M. de Castro e Sousa, S. M. M. de Moura Dantas, H. M. L. Rolim, A. A. de Carvalho Melo-Cavalcante, M. S. Mubarak, N. S. Yarla, J. A. Shilpi, S. K. Mishra, A. G. Atanasov and M. A. Kamal, Cancer Lett., 2018, 420, 129-145.

3 N. P. Trivedi, U. M. Rawal and B. P. Patel, Integr. Cancer Ther., 2007, 6, 271.

4 N. K. Pushpendra Kumar Jain, Y. Pounikar, A. Gajbhiye and M. D. Kharya, J. Liposome Res., 2013, 23, 110-118.

5 P. Roy, S. Das, T. Bera, S. Mondol and A. Mukherjee, Int. J. Nanomed., 2010, 5, 1113-1121.

6 B. A. Aishwarya Balap, S. Lohidasan and K. M. Arulmozhi Sinnathambi, J. Ethnopharmacol., 2016, 183, 9-17.

7 A. P. P. Bothiraja Chellampillai, Int. J. Nanotechnol., 2011, 8, 764-778.
8 J. J. Zou, G. U. Xiao-Xiang, Y. B. Zhu, H. W. Fan and D. W. Xiao, Chin. J. Pharm., 2007, 38, 506-964.

9 J. Chang, R. M. Zhang, Y. Zhang, Z. B. Chen, Z. M. Zhang, Q. Xu, Y. P. Yang, Y. Y. Long, L. L. Liu and H. Y. Cai, J. Chin. Integr. Med., 2008, 6, 1238-1245.

10 D. U. Hong, X. Niu, Q. Feng and Y. E. Minglei, Traditional Chinese Drug Research \& Clinical Pharmacology, 2007.

11 F. J. A. Rabea Parveen, Z. Iqbal, M. Samim and S. Ahmad, Drug Dev. Ind. Pharm., 2014, 40(9), 1206-1212.

12 Q. J. Ping Du, R. Yang, C. Liu, Y. Li, a L. Wang, P. Xue, Q. Fu and T. Zhang, RSC Adv., 2016, 6, 101404-101414.

13 L. Z. Tongtong Zhang, M. Li, Y. Hu, E. Zhang, Q. Jiang, G. Han and Y. Jin, Mol. Pharm., 2017, 14(5), 1718-1725.

14 E. Wenk, H. P. Merkle and L. Meinel, J. Controlled Release, 2011, 150, 128-141.

15 T. Yucel, M. L. Lovett and D. L. Kaplan, J. Controlled Release, 2014, 190, 381-397.

16 B. Subia and S. C. Kundu, Nanotechnology, 2013, 24, 035103.

17 J. Qu, Y. Liu, Y. Yu, J. Li, J. Luo and M. Li, Mater. Sci. Eng., C, 2014, 44, 166-174.

18 M. Chen, Z. Shao and X. Chen, J. Biomed. Mater. Res., 2012, 100, 203-210.

19 V. G. Anshu B Mathur, Nanomedicine, 2010, 5, 807-820.

20 E. Wenk, A. J. Wandrey, H. P. Merkle and L. Meinel, J. Controlled Release, 2008, 132, 26-34.

21 H.-L. W. Jiang Liu, J. Moselhy, B. Bowen, X. Y. Wu and M. R. Johnston, Lung Cancer, 2006, 51, 377-386.

22 D. M. Jiang Liu, E. Kwong, X. Y. Wu and M. R. Johnston, Biomaterials, 2007, 28, 3236-3244.

23 X. W. Guotao Cheng, S. Tao, J. Xia and S. Xu, J. Appl. Polym. Sci., 2015, 132(22), 1-8.

24 M. M. Tomonori Waku, T. Kaneko and M. Akashi, Macromolecules, 2007, 40, 6385-6392.

25 L. Z. Juliana, M. Chan, K. P. Yuet, G. Liao, J.-W. Rhee, R. Langer and O. C. Farokhzad, Biomaterials, 2009, 30, 1627-1634.

26 C. Yan, B. Yang and Z. Yu, Anal. Methods, 2014, 6, 248-252. 27 S. Yu, W. Yang, S. Chen, M. Chen, Y. Liu, Z. Shao and X. Chen, RSC Adv., 2014, 4, 18171.

28 N. Drnovsek, R. Kocen, A. Gantar, M. Drobnic-Kosorok, A. Leonardi, I. Krizaj, A. Recnik and S. Novak, J. Mater. Chem. B, 2016, 4, 6597-6608. 